\title{
Intrapersonal Mindfulness is Associated with Reduced Risk of Burnout among Central Appalachian Educators
}

\author{
Dr. Chris Anama-Green, EdD, MPH, MAT
}

\begin{abstract}
\section{Introduction}

National statistics suggest that up to $40 \%$ of new teachers will leave their school or the teaching profession within their first five years of teaching. Much of this attrition is associated with work-related burnout, some of which may be preventable with targeted worksite health interventions. Previous research suggests that mindfulness skills may be protective from burnout, ultimately reducing the likelihood of attrition from the profession.
\end{abstract}

\section{Methods}

This study compared the self-reported levels of burnout and secondary traumatic stress with participants' levels of interpersonal and intrapersonal mindfulness. Participants completed the Professional Quality of Life Inventory and the Mindfulness in Teaching Inventory. Odds Ratios and Relative Risks were calculated using Epi Info. Pearson correlations, linear regression, and ANOVA analyses were completed using SPSS. Chronbach's alpha values were also calculated to evaluate score reliability of the five constructs.

\section{Results}

Relative Risks and Odds Ratios of having secondary traumatic stress scores of "average/high" were significantly lower for those with high intrapersonal mindfulness scores. Those who reported high intrapersonal mindfulness scores were up to 11 times more likely to report "low" burnout than those who reported low intrapersonal mindfulness scores. Burnout negatively correlated with both intrapersonal mindfulness suggesting that as intrapersonal mindfulness level decreases, burnout increases. ANOVA testing identified significant differences in burnout based on intrapersonal mindfulness level.

\section{Conclusion}

Those who reported high levels of intrapersonal mindfulness had significantly reduced risk of burnout. These results will inform further research in the region regarding mindfulness practice and the experience of burnout among teachers in the region. Thus, mindfulness may be protective from occupation-related burnout for this population. Interventions informed by additional research could reduce the burden of occupation-related burnout and may ultimately contribute toward reduced attrition in the teaching profession. 


\section{Background}

Previous research related to mindfulness in education suggests that mindfulness practices, such as Mindfulness-Based Stress Reduction (MBSR) and others, are associated with numerous physical health and psychosocial benefits for both K-12 teachers and students. (Frank et al., 2016; Harris et al., 2016) Recent research suggests that mindfulness practices may be associated with reduced teacher burnout, which could contribute to reduced attrition from the profession over time.

While large-scale national studies have identified factors associated with teacher attrition in the United States, (Raue \& Gray, 2015) studies regarding teacher attrition in Central Appalachia are sparse. Further, limited research has been published regarding epidemiologic factors related to K-12 teacher mental health in the Central Appalachian region. If the region's educational culture is consistent with national trends, attrition may be in part due to work-related mental health problems including occupational burnout, "Compassion Fatigue" associated with "helping" professions, and elevated anxiety and depression (Stamm, 2010).

\section{Burnout}

Several studies have linked stress and burnout with employee turnover. A study of the effects of stress on teachers by the Robert Wood Johnson Foundation and Pennsylvania State University attributed the high turnover rate in teaching in part to job-related stress. The same report noted that this loss of teachers results in high costs for districts and states, negative impacts on student achievement, and "destabilization" in school-community relations in low-resource communities (Greenberg et al., 2016). Other studies in the United States and abroad (Lauermann \& König, 2016; Luken \& Sammons, 2016) have also described the relationship between high job-related stress leading to burnout and teacher attrition, particularly in difficult-to-fill subjects (Goldhaber et al., 2016) and low-income communities (Cowan et al., 2016).

As burnout becomes a widespread problem for a specific group of people, it becomes a public health problem. Given the common co-morbidity of burnout with a host of physical and mental health conditions documented in literature (Koutsimani et al., 2019), burnout can be studied as a health syndrome (Salvagioni et al., 2017). Some assert that burnout is a diagnosable medical or mental health condition (Bianchi et al., 2015; Schonfeld \& Bianchi, 2016). In any case, the severity of burnout reported nationally by teachers (Ryan et al., 2017) coupled with the steady turnover (Greenberg et al., 2016) in the profession suggests a sufficiently high prevalence of burnout for analysis using epidemiological methods.

Odds Ratio (Szumilas, 2010) and Relative Risk (Tenny \& Hoffman, 2019) are common measures used in epidemiology to estimate risk and to understand association between risk factors and disease states. These measures can be used to understand the "risk factors" associated with manifestation of burnout or the "disease state." These methods can also identify protective factors from disease states (Szumilas, 2010). Thus, the role of 
mindfulness in preventing, reducing, or even promoting burnout can be identified and conceptualized using Odds Ratios and Relative Risk.

\section{Mindfulness}

Mindfulness refers to a set of practices that decades of research has linked with positive health and psychosocial benefits (Harris et al., 2016; Jennings et al., 2017). Though mindfulness originated as a religious practice, contemporary mindfulness interventions implemented in clinical settings and in schools use secular evidence-based mindfulness practices (Frank et al., 2013).

Key benefits of mindfulness are those associated with stress reduction and emotional regulation (Jennings et al., 2017). Reduced stress and improved capacity to regulate one's emotions leads to happier employees and a more productive workplace (Luken \& Sammons, 2016). A workplace mindfulness framework published in 2016 describes this relationship in context with current research. According to the authors, mindfulness practice at work strengthens attention. As a result, cognitive fitness is improved and physiological response to stress while enhancing self-regulation of behavior and emotion. This leads to a plethora of positive workplace outcomes organized under the themes of increased performance, stronger relationships, and enhanced well-being (Good et al., 2016). Other published research studies also support these ideas (Chin et al., 2018; Jennings et al., 2017; Luken \& Sammons, 2016).

A systematic review of workplace mindfulness practices indicated that mindfulness was effective in reducing symptoms of burnout in teachers according to current published research (Luken \& Sammons, 2016). Researchers in other studies have alluded to the idea that mindfulness training may reduce symptoms of job-related burnout among educators (Frank et al., 2016). The "CARE for Teachers" intervention, which is delivered as professional development for teachers, was reported to improve emotional regulation and fewer symptoms of "psychological distress" (Jennings et al., 2017). Another study of CARE for Teachers reported that teacher participation in the program heightened teacher awareness about the importance of self-care and was associated with reduced reactivity to emotionally-charged situations (Schussler et al., 2016). A systematic review of mindfulness programs in schools suggested that mindfulness' effect on improving resilience may be associated with its association in decreased work-related stress (Zenner et al., 2014).

Although published literature provides strong support for the implementation of mindfulness interventions to support teachers' psychosocial and physical health (RuijgrokLupton et al., 2018), the body of mindfulness intervention literature focuses on teacher populations outside of central Appalachia. Further, while the possible association between elevated mindfulness skill-level and reduced burnout in teachers has been proposed (Frank et al., 2016; Jennings et al., 2017; Luken \& Sammons, 2016), the idea had not been tested with central Appalachian educators. As the first step in assessing the role of mindfulness skills in central Appalachian educators, this study was designed to provide insight regarding the potential for future mindfulness intervention use with educators from the region. Specifically, the relationship identified between mindfulness skill-level and burnout 
will support mindfulness intervention studies in central Appalachia to test the potential for mindfulness training as a means to reduce burnout.

\section{Methods}

This study employed quantitative methodology for survey administration and analysis. Participants completed two inventories and a brief demographic survey. Survey instruments used in this study included the Mindfulness in Teaching Scale (Frank et al., 2016) and the Professional Quality of Life Inventory (ProQOL) (Stamm, 2010).

Demographic questions included age, gender, years working in education, years working in current district, role in education, grade(s) taught, and level of education. Items from both the Mindfulness in Teaching Scale and ProQOL were Likert-scale questions. Demographic questions were multiple choice or "select all that apply" questions. The two instruments and the demographic questions were delivered online.

\section{Sampling Procedure}

Educators in the Eastern Kentucky counties of the central Appalachian region were invited to participate in this study through professional learning networks, regional educational cooperatives, and professional education organizations. Invitations to participate were provided via email. Informed consent was collected electronically before participants begin the survey.

\section{Analysis}

The ProQOL subscale scores for burnout and secondary traumatic stress were calculated in accordance with the instructions in the ProQOL manual. Five items on the burnout subscale were reversed before the burnout score was calculated. Because the ProQOL is a standardized inventory, these scores can be interpreted individually and aggregately using score values provided in the ProQOL manual in addition to the statistical analyses provided in this paper (Stamm, 2010).

Educator mindfulness scores from the Mindfulness in Teaching scale (Frank et al., 2016) were calculated in two subscales, which included Interpersonal Mindfulness and Intrapersonal Mindfulness. Intrapersonal mindfulness responses were "reverse coded" before calculations were completed. Each subscale score was be used in comparisons with self-reported burnout and other variables.

Odds Ratios and Relative Risks were calculated for each dimension of mindfulness and secondary traumatic stress and burnout using Epi Info. The Haldane-Anscombe correction was applied prior to analysis for any table values below zero (Ruxton \& Neuhaüser, 2012). Confidence intervals were calculated for use as a surrogate for significance testing when interpreting Odds Ratios and Relative Risks (Szumilas, 2010).

SPSS was used for all remaining statistical tests. Full descriptive analysis was conducted for demographic variables to summarize and describe the research participant population. ANOVA tests and Pearson correlations among the subscale constructs were performed. 
Chronbach's Alpha values were also calculated to confirm score reliability of the five constructs (Mallery \& George, 2003).

\section{Results}

Out of a total of 144 participants, most $(\mathrm{n}=113,74.3 \%)$ were female. Across age categories, $\mathrm{n}=8(5.3 \%)$ participants were between $20-25, \mathrm{n}=32(21.1 \%)$ were between 26-35, $(29.6 \%)$ were between $36-45, \mathrm{n}=44(28.9 \%)$ were between $46-55$, and the remainder of $\mathrm{n}$ $=15(9.9 \%)$ were between 56 and 65 . The majority of participants $(n=140,92.1 \%)$ reported their race as White/Caucasian and the remaining reported two or more races $(\mathrm{n}=2,1.3 \%)$, "Asian or Pacific Islander" $(\mathrm{n}=1)$, and "other" $(\mathrm{n}=1)$. No participants reported Hispanic or Latino ethnicity.

For grade levels taught, $n=48(31.6 \%)$ taught elementary grades only, $n=21(13.8 \%)$ taught middle grades only, $\mathrm{n}=36(23.7 \%)$ reported high school grades only, $\mathrm{n}=22$ $(14.5 \%)$ reported elementary and middle grades, $\mathrm{n}=10(6.6 \%)$ reported middle and high school grades, and $\mathrm{n}=5(3.3 \%)$ taught all grades.

Chronbach's Alpha coefficients were calculated for each scale and interpreted using criteria from Mallery and George (2003). The Alpha values rounded to two decimal points were as follows: Intrapersonal Mindfulness Alpha $=0.82$; Interpersonal Mindfulness Alpha $=0.70$; burnout Alpha $=0.80$; and Secondary Traumatic Stress Alpha $=0.79$. The Interpersonal Mindfulness alpha value is in the "acceptable" range. The Secondary Traumatic Stress alpha value is "acceptable" approaching "good." Intrapersonal Mindfulness and burnout alpha values are "good." Overall, these scores indicate that the scales are of sufficient reliability for use with this study population.

Pearson correlations were calculated for burnout, secondary traumatic stress (STS), intrapersonal mindfulness, and interpersonal mindfulness. Results are displayed in Table 1. Burnout and STS were positively correlated suggesting that as burnout increases, Secondary Traumatic Stress also increases $(r[142]=0.642, p<.05)$. Burnout also negatively correlated with both Intrapersonal Mindfulness suggesting that as Intrapersonal Mindfulness skill level decreases, burnout increases ( $r[142]=-.616, p<.05)$. A weak negative correlation was also found between burnout and Interpersonal Mindfulness $(r$ $[142]=-.227, p<.05)$.

Secondary traumatic stress had a negative correlation with Intrapersonal Mindfulness $(r$ $[142]=0 .-523, p<.05)$. This indicates a similar relationship as with burnout and Intrapersonal Mindfulness. Almost no measurable correlation was present between Interpersonal Mindfulness and Secondary Traumatic Stress ( $r[142]=0.18, p>.05)$. Interpersonal mindfulness and Intrapersonal Mindfulness had a weak positive correlation $(r[142]=0.192, p>.05)$. This is referenced in Table 1 .

Table 1. Pearson Correlations for all Subscales 


\begin{tabular}{|c|c|c|c|}
\hline & $\underline{\text { STS }}$ & $\begin{array}{l}\text { Intrapersonal } \\
\text { Mindfulness }\end{array}$ & $\begin{array}{l}\text { Interpersonal } \\
\text { Mindfulness }\end{array}$ \\
\hline Burnout & $.642 *$ & $-.616^{*}$ & $-.227^{*}$ \\
\hline $\begin{array}{l}\text { Secondary } \\
\text { Traumatic } \\
\text { Stress }\end{array}$ & & $-.523^{*}$ & .018 \\
\hline $\begin{array}{l}\text { Intrapersonal } \\
\text { Mindfulness }\end{array}$ & & & $.192 *$ \\
\hline
\end{tabular}

Notes. $* p<.05$, two-tailed.

An ANOVA analysis was conducted for Intrapersonal Mindfulness with burnout as the dependent variable. Because only one case was present for score averages of 1.0 or below, Intrapersonal Mindfulness scores were recoded into three categories. Burnout scores were also recoded into three categories, in accordance with those described in the ProQOL manual (Stamm, 2010). Results for the ANOVA test of burnout and Intrapersonal Mindfulness were significant with $F[2]=8.928, p<.05$. This suggests that significant differences are present in reported level of burnout depending on reported level of Intrapersonal Mindfulness. See Table 2.

Table 2. ANOVA Intrapersonal Mindfulness by Burnout Level

\begin{tabular}{llllll}
\hline & $\underline{\text { Sum of }}$ & $\underline{\text { df }}$ & $\underline{\text { Mean Square }}$ & $\underline{\text { F }}$ & $\underline{\text { Sig. }}$ \\
\hline Between & .749 & 2 & .374 & 8.928 & 0.000 \\
Groups & & & & & \\
Within & 5.911 & 141 & .042 & & \\
Groups & & & & & \\
Total & 6.660 & 143 & & & \\
\hline
\end{tabular}

Relative Risks and Odds Ratios of reporting "low" burnout for those with Intrapersonal Mindfulness scores of 3+ or 4+ were significantly higher than 1.0. These statistics did not contain 1.0 within their confidence intervals and were significant at the $p<.05$ level. This indicates that those who reported Intrapersonal Mindfulness scores of greater than $3+$ and greater than $4+$ were approximately 6 times more likely $(\mathrm{OR}=6.79, \mathrm{CI}=1.95-23.66)$ and 11 times more likely $(\mathrm{OR}=11.58, \mathrm{CI}=5.06-26.52)$ to report "low" burnout than those who reported Intrapersonal Mindfulness scores below 3. Interpersonal Mindfulness risk and odds calculations were not significant, precluding determination of the risk and odds relationships between Interpersonal Mindfulness and burnout.

Participants with Intrapersonal Mindfulness scores of $3+(\mathrm{OR}=0.15, \mathrm{CI}=0.04-0.51)$ and $4+$ $(\mathrm{OR}=0.10, \mathrm{CI}=0.40-0.22)$ had reduced risks and odds of reporting "Average/High" burnout as compared with those reporting Intrapersonal Mindfulness scores below 3 . Again, Interpersonal Mindfulness Odds Ratios and Relative Risks were not significant. This prevents valid interpretation of the relationship between Interpersonal Mindfulness and "Average/High" burnout. Future research will be required to understand the relationship between Interpersonal Mindfulness and burnout. (See Table 3.) 


\section{Discussion}

Relative Risks and Odds Ratios between intrapersonal mindfulness and both burnout and secondary traumatic stress, respectively, suggest that intrapersonal mindfulness in the classroom may be a protective factor for teachers in terms of reducing the risk of burnout and STS. Those with higher intrapersonal mindfulness scores have reduced risk for high levels of burnout and increased risk for low levels of burnout. This is consistent with previous findings on mindfulness in education. ${ }^{1,2}$ However, level of Interpersonal Mindfulness did not significantly alter the risk of any level of burnout. More research is recommended to better understand this as well as other factors that may affect both a teacher's risk of burnout and mindfulness level.

The results for Intrapersonal Mindfulness and burnout mirror findings from intervention studies conducted with teachers in other areas of the United States, which indicated decreased feelings of burnout for teachers involved in mindfulness practices (Jennings et al., 2013, 2017). With the understanding that this pattern holds true among Central Appalachian educators, the translation of these findings into practice become an important next step for educational leaders in the region.

\section{Limitations}

Data collection for this study was limited to a "single point in time," which may not have been representative of all aspects of the participants' normal work environments. Collection at multiple points in the year could provide a more comprehensive and realistic view of participants' experiences with mindfulness and burnout in the classroom. Further, the survey instrument for this study was administered at the end of an academic year, which may have affected teacher perceptions about burnout and mindfulness as compared with other points of time within a typical school year.

Participants volunteered to be included in the study meaning that those drawn to the ideas of burnout and/or mindfulness may have been more likely to participate. This could affect responses given, particularly with respect to any existing beliefs or opinions about one's level of burnout or mindfulness at the time of the survey. Further, because participants were not asked about their participation in mindfulness practice, mindfulness, integrative stress management, or other self-care strategies, this study prohibits an understanding of the underlying practices that could have affected some responses.

Sample demographics did not permit for comparison by race as too few racial and ethnic minority teachers participated in the study. This precludes the interpretation of these results for teachers from racial and ethnic minority populations in Eastern Kentucky as these results may not mirror their experiences. Future studies on this topic in Eastern Kentucky and in Appalachia should seek out minority teachers to accurately and inclusively understand their experiences about mindfulness and burnout.

Limitations associated with mindfulness research apply to any study that attempts to quantify mindfulness. Because scholars do not have a universally accepted definition of 
mindfulness, interpretation of any research on the topic is subject to the confines of the definition used by any given study. As such, comparability of results between and among studies is somewhat limited (Davidson \& Kaszniak, 2015). The mindfulness instrument in this study, the Mindfulness in Teaching scale was designed specifically for use with teachers (Frank et al., 2016). However, results from studies that rely on other conceptualizations of mindfulness may not be comparable with the findings from this research.

\section{Implications for Future Study}

While this project provided a baseline understanding about the experience of mindfulness for teachers in the region, the study did not reveal any information about the mindfulness practices of teachers in the region. However, understanding what mindfulness practices, if any, in which teachers engage now becomes an important consideration in making broader interpretations about the findings and recommending interventions. Future studies on the topic in the region should specifically focus on personal mindfulness practices as well as those that may be provided by individual school districts. This could inform a future pilot study in which one or more mindfulness interventions could be used to reduce the burden of burnout on teachers and schools.

Results from this study summarized general findings regarding the experience of burnout and the two dimensions of mindfulness in the population of interest. However, beyond demographic information and the subscales of the ProQOL instrument, no specific indicators regarding factors that cause or modulate burnout and mindfulness in teachers were identified. A study focused on identifying and understanding these factors would be useful for selecting interventions to address burnout and evaluating educational system components associated with burnout.

An important consideration to help understand the larger picture of mindfulness-related traits lies in how they are cultivated. A question worth considering is "what factors or activities, other than mindfulness practices, create mindfulness-like skills?" It is possible that one could receive a high mindfulness score without having practiced mindfulness. In other words, it may be possible to develop the traits and skills measured by mindfulness inventories without a traditional mindfulness practice. Identifying these factors, if any exist, would be helpful in addressing the problem of burnout among educators in the region.

Multiple analyses suggested a lack of connection between interpersonal mindfulness and burnout, despite the repeatedly demonstrated strong connection between intrapersonal mindfulness and burnout. Considering the case of Interpersonal Mindfulness is important for understanding the overall relationship between mindfulness and teacher burnout. Given that interpersonal mindfulness refers to social interactions that may be subject to cultural mores or taboos, these hidden variables may play a part in influencing "socially acceptable" behavior (Davidson \& Kaszniak, 2015; Farias \& Wikholm, 2016; Van Dam et al., 2018). This includes a teacher's behavior in a school and classroom. Therefore, the 
measure of Interpersonal Mindfulness may be an accurate interpretation of a teacher's social behavior without being a fair depiction of the degree to which mindfulness influences that behavior. This concern for social acceptability could cause teacher behavior to mimic mindfulness-informed interactions even while the teacher experiences a high level of burnout. Research to understand the relationship between social acceptability and interpersonal mindfulness may help us understand why it is not a predictor of burnout while its counterpart intrapersonal mindfulness is a strong predictor of burnout.

\section{Conclusion}

Teacher intrapersonal mindfulness in a classroom setting may be a protective factor for teachers by reducing the risk of burnout and secondary traumatic stress. The role of interpersonal mindfulness remains unclear. Further research can help us to better understand the relationship between interpersonal mindfulness and burnout. Results from this study will inform further research to reduce teacher attrition in the region. 


\section{References}

Bianchi, R., Schonfeld, I. S., \& Laurent, E. (2015). Is burnout separable from depression in cluster analysis? A longitudinal study. Social Psychiatry and Psychiatric Epidemiology, 50(6), 1005-1011. https://doi.org/10.1007/s00127-014-0996-8

Chin, B., Slutsky, J., Raye, J., \& Creswell, J. D. (2018). Mindfulness training reduces stress at work: A randomized controlled trial. Mindfulness. https://doi.org/10.1007/s12671018-1022-0

Cowan, J., Goldhaber, D., Hayes, K., \& Theobald, R. (2016). Missing elements in the discussion of teacher shortages. Educational Researcher, 45(8), 460-462. https://doi.org/10.3102/0013189X16679145

Davidson, R. J., \& Kaszniak, A. W. (2015). Conceptual and methodological issues in research on mindfulness and meditation. American Psychologist, 70(7), 581-592. https://doi.org/10.1037/a0039512

Farias, M., \& Wikholm, C. (2016). Has the science of mindfulness lost its mind? BJPsych Bulletin, 40(6), 329-332. https://doi.org/10.1192/pb.bp.116.053686

Frank, J. L., Jennings, P. A., \& Greenberg, M. T. (2013). Mindfulness-Based Interventions in School Settings: An Introduction to the Special Issue. Research in Human Development, 10(3), 205-210. https://doi.org/10.1080/15427609.2013.818480

Frank, J. L., Jennings, P. A., \& Greenberg, M. T. (2016). Validation of the Mindfulness in Teaching Scale. Mindfulness, 7(1), 155-163. https://doi.org/10.1007/s12671-0150461-0

Goldhaber, D., Krieg, J., Theobald, R., Brown, N., Goldhaber, B. D., Krieg, J., Theobald, R., \& Brown, N. (2016). Refueling the STEM and special education teacher pipelines. Kappan, 97(4), 56-62. https://doi.org/10.1177/0031721715619921

Good, D. J., Lyddy, C. J., Glomb, T. M., Bono, J. E., Brown, K. W., Duffy, M. K., Baer, R. A., Brewer, J. A., \& Lazar, S. W. (2016). Contemplating mindfulness at work: An integrative review. Journal of Management, 42(1), 114-142. https://doi.org/10.1177/0149206315617003

Greenberg, M. T., Brown, J. L., \& Abenavoli, R. M. (2016). Teacher stress and health: Effects on teachers, students, and schools. In Edna Bennett Pierce Prevention Research Center, The Pennsylvania State University (Issue September 2016). https://www.rwjf.org/content/dam/farm/reports/issue_briefs/2016/rwjf430428\%0 Ahttp://www.rwjf.org/en/library/research/2016/07/teacher-stress-and-health.html

Harris, A. R., Jennings, P. A., Katz, D. A., Abenavoli, R. M., \& Greenberg, M. T. (2016). Promoting stress management and wellbeing in educators: Feasibility and efficacy of a school-based yoga and mindfulness intervention. Mindfulness, 7(1), 143-154. https://doi.org/10.1007/s12671-015-0451-2

Jennings, P. A., Brown, J. L., Frank, J. L., Doyle, S., Oh, Y., Davis, R., Rasheed, D., DeWeese, A., DeMauro, A. A., Cham, H., \& Greenberg, M. T. (2017). Impacts of the CARE for teachers program on teachers' social and emotional competence and classroom interactions. Journal of Educational Psychology, 109(7), 1010-1028. https://doi.org/10.1037/edu0000187

Jennings, P. A., Frank, J. L., Snowberg, K. E., Coccia, M. A., \& Greenberg, M. T. (2013). Improving classroom learning environments by cultivating awareness and resilience in education (CARE): Results of a randomized controlled trial. School Psychology 
Quarterly, 28(4), 374-390. https://doi.org/10.1037/spq0000035

Koutsimani, P., Montgomery, A., \& Georganta, K. (2019). The relationship between burnout, depression, and anxiety: A systematic review and meta-analysis. Frontiers in Psychology, 10(MAR), 1-19. https://doi.org/10.3389/fpsyg.2019.00284

Lauermann, F., \& König, J. (2016). Teachers' professional competence and wellbeing: Understanding the links between general pedagogical knowledge, self-efficacy and burnout. Learning and Instruction, 45, 9-19. https://doi.org/10.1016/j.learninstruc.2016.06.006

Luken, M., \& Sammons, A. (2016). Systematic review of mindfulness practice for reducing job burnout. The American Journal of Occupational Therapy, 70(2). https://doi.org/10.5014/ajot.2016.016956

Mallery, P., \& George, D. (2003). SPSS for Windows step by step: a simple guide and reference. Allyn, Bacon.

Raue, K., \& Gray, L. (2015). Public school teacher attrition and mobility in the first five years: Results from the first through fifth waves of the 2007-08 Beginning Teacher Longitudinal Study. National Center for Education Statistics, 1-40. https://doi.org/Article

Ruijgrok-Lupton, P. E., Crane, R. S., \& Dorjee, D. (2018). Impact of mindfulness-based teacher training on MBSR participant well-being outcomes and course satisfaction. Mindfulness, 9(1), 117-128. https://doi.org/10.1007/s12671-017-0750-x

Ruxton, G. D., \& Neuhaüser, M. (2012). Review of alternative approaches to calculation of a confidence interval for the odds ratio of a 232 contingency table. https://doi.org/10.1111/j.2041-210x.2012.00250.x

Ryan, S. V, von der Embse, N. P., Pendergast, L. L., Saeki, E., Segool, N., \& Schwing, S. (2017). Leaving the teaching profession: The role of teacher stress and educational accountability policies on turnover intent. Teaching and Teacher Education, 66, 1-11. https://doi.org/10.1016/j.tate.2017.03.016

Salvagioni, D. A. J., Melanda, F. N., Mesas, A. E., González, A. D., Gabani, F. L., \& De Andrade, S. M. (2017). Physical, psychological and occupational consequences of job burnout: A systematic review of prospective studies. PLOS ONE, 12(10), 1-29. https://doi.org/10.1371/journal.pone.0185781

Schonfeld, I. S., \& Bianchi, R. (2016). Burnout and depression: Two entities or one? Journal of Clinical Psychology, 72(1), 22-37. https://doi.org/10.1002/jclp.22229

Schussler, D. L., Jennings, P. A., Sharp, J. E., \& Frank, J. L. (2016). Improving teacher awareness and well-being through care: A qualitative analysis of the underlying mechanisms. Mindfulness, 7(1), 130-142. https://doi.org/10.1007/s12671-015-04227

Stamm, B. H. (2010). The Concise ProQOL Manual (2nd ed.). ProQOL.org.

Szumilas, M. (2010). Explaining Odds Ratios. Journal of the Canadian Academy of Child and Adolescent Psychiatry, 19(3), 227-229. https://doi.org/10.1136/bmj.c4414

Tenny, S., \& Hoffman, M. R. (2019). Relative Risk. https://www.ncbi.nlm.nih.gov/books/NBK430824/

Van Dam, N. T., van Vugt, M. K., Vago, D. R., Schmalzl, L., Saron, C. D., Olendzki, A., Meissner, T., Lazar, S. W., Kerr, C. E., Gorchov, J., Fox, K. C. R. R., Field, B. A., Britton, W. B., Brefczynski-Lewis, J. A., \& Meyer, D. E. (2018). Mind the hype: A critical evaluation and prescriptive agenda for research on mindfulness and meditation. Perspectives on 
Psychological Science, 13(1), 36-61. https://doi.org/10.1177/1745691617709589 Zenner, C., Herrnleben-Kurz, S., \& Walach, H. (2014). Mindfulness-based interventions in schools-A systematic review and meta-analysis. Frontiers in Psychology, 5, 1-20. https://doi.org/10.3389/fpsyg.2014.00603 